\title{
INTERACTING MULTIPLE MODEL ALGORITHM FOR MANOEUVRING SHIP TRACKING BASED ON NEW SHIP MODELS
}

\section{Emil SEMERDJIEV, Ludmila MIHAYLOVA, Tzvetan SEMERDJIEV and Violeta BOGDANOVA}

\section{Introduction}

Tracking of manoeuvring targets is a problem of a great practical and theoretical interest. The real-world tracking applications meet a number of difficulties caused by the presence of different kinds of uncertainty due to the unknown or not precisely known system model and random processes' statistics or because of their abrupt changes. $^{2-5,9}$ These problems are especially complicated in the marine navigation practice, $^{7}, 14,15,19$ where the commonly used simple models of rectilinear or curvilinear target motions do not match the highly non-linear dynamics of the manoeuvring ship. A solution of these problems is to derive more adequate descriptions of the real ship dynamics and to design adaptive estimation algorithms.

Such a solution is proposed in the paper. A new ship model is derived in Section 2 after an analysis of the basic hydrodynamic models. The derived model is implemented in a new version of the Interacting Multiple Model (IMM) tracking algorithm - the most cost-effective multiple model algorithm for hybrid estimation. ${ }^{3,6}$, 10, 11 The proposed model and tracking algorithm are presented in Section 3 and evaluated in Section 4.

\section{Model identification}

Results of a study, described in ${ }^{16,17,18}$ are summarised in this section. It should be noted that the high complexity of the hydrodynamic processes caused by the ship motion in deep and confined water and the wide variety of ship forms and sizes lead to various non-stochastic ship models. These models can be divided in two groups: precise models, topical for particular ship forms and sizes (the Sobolev, ${ }^{19}$ Cubic, ${ }^{1}$ Quadratic $^{13}$ and $\mathrm{MMG}^{14}$ models) and models with greater generality but lower 
accuracy (the Pershitz ${ }^{15}$ and Nomoto ${ }^{12}$ models). Here, the widely used continuoustime (CT) Pershitz model ${ }^{15}$ is chosen as a basic model to assure a good trade-off between complexity and accuracy:

$$
\begin{aligned}
& \frac{d X}{d t}=K_{V} V_{0} \sin (\psi-\beta), \\
& \frac{d Y}{d t}=K_{V} V_{0} \cos (\psi-\beta), \\
& \frac{d \psi}{d t}=K_{V} \omega, \\
& \frac{d \omega}{d t}=-\left(\frac{V_{0}}{L}\right)^{2}\left(q_{31} \beta+s_{31} \delta\right)-\frac{V_{0}}{L} r_{31} \omega, \\
& \frac{d \beta}{d t}=-\frac{V_{0}}{L}\left(q_{21} \beta+h_{1} \beta|\beta|+s_{21} \delta\right)-r_{21} \omega, \\
& V=V_{0} K_{V} ; \\
& K_{V}=\frac{V(\omega)}{V(0)}=\frac{V}{V_{0}}=\left(1+1.9 \omega^{2} L^{2} V_{0}^{-2}\right)^{-1} \leq 1 .
\end{aligned}
$$

The state vector of the considered CT model is $x=[X, Y, \psi, \omega, \beta, V]^{\prime}$. It includes the ship coordinates and heading, rate of turn, drift angle and velocity; $\delta$ is the control rudder angle deviation. The constants $q_{21}, r_{21}, s_{21}, h_{1}, q_{31}, r_{31}$ and $s_{31}$ are hydrodynamic coefficients depending on the ship geometry, most of all, and on the ship length $L .^{20}$ Equations (3) and (6) illustrate the main feature of the considered dynamics - the non-linear dependence between the rate of turn and the velocity of the ship. This is the main difference between the presented model in this paper and other well-known simple models., 5,9

Very often in the available literature sources ${ }^{15,20}$ this model is simplified by substituting the factor $|\beta|$ with an off-line computed factor:

$$
\beta_{0}=\frac{-q+\sqrt{q^{2}+4 h_{1} r_{31} s|\delta|}}{2 h_{1} r_{31}},
$$


where: $q=q_{21} r_{31}-q_{31} r_{21}, s=r_{21} s_{31}-r_{31} s_{21}$. Then, the system of two first-order differential equations consisting of equation (4) and the modified equation (5) is transformed in two independent second-order differential equations, omitting the negligible second-order derivatives:

$$
\begin{aligned}
& 2 p \frac{d \omega}{d t} \frac{L^{2}}{V_{0}^{2}}+q \omega \frac{L}{V_{0}}+s_{31} \delta=0 ; \\
& 2 p \frac{d \beta}{d t} \frac{L}{V_{0}}+q \beta+s_{21} \delta=0,
\end{aligned}
$$

where $p=0.5\left(q_{21}^{*}+r_{31}\right), q^{*}=q_{21}^{*} r_{31}-q_{31} r_{21}, q_{21}^{*}=q_{21}+h_{1} \beta_{0}$. The final CT model (1)-(3), (4') and (6) is obtained by setting $\beta \equiv 0$.

The respective discrete-time (DT) model is:

$$
\begin{aligned}
& X_{k+1}=X_{k}+T V_{k} \sin \psi_{k}, \\
& Y_{k+1}=Y_{k}+T V_{k} \cos \psi_{k}, \\
& \left.\psi_{k+1}=\psi_{k}+T V_{k} \Omega_{k}+0.5 T \tau V_{k}\left(\Omega_{k}-\Omega_{0}\right) e^{T V_{k} \tau}\right], \\
& \Omega_{k+1}=\Omega_{k} e^{T V_{k} \tau}+\Omega_{0}\left(1-e^{T V_{k} \tau}\right), \\
& V_{k}=V_{0} K_{V}=V_{0}\left(1+1.9 \Omega_{k}^{2} L^{2}\right)^{-1},
\end{aligned}
$$

where

$$
\begin{gathered}
\tau=\frac{-0.5 p+\sqrt{0.25 p^{2}-q^{*}}}{L}, \quad\left[m^{-1}\right], \\
\Omega_{0}=\frac{\omega}{V_{0}}=-\frac{\left[s_{31} \delta+\operatorname{sign}(\delta) q_{31} \beta_{0}\right]}{r_{31} L},\left[\frac{r a d}{m}\right] .
\end{gathered}
$$

and $k=1,2, \cdots ; T$ is the sampling interval. 
The full coincidence between the results obtained by the CT model (1)-(6), and these obtained by the derived DT model (7)-(11) is demonstrated in ${ }^{17}$. Model (7)-(11) is used for true data generation in further simulations.

The final DT model, suitable for implementation in Kalman filter, is composed on the basis of the assumptions ${ }^{17,18}$ :

* It is assumed that the observed ship maneuvers with a constant rate of turn:

$$
\Omega_{k+1}=\Omega_{k} \quad \text { (i.e. } \tau \equiv 0 \text { ). }
$$

* The whole domain of unknown control parameters $\Omega_{k}$ is replaced by a set of three control parameters corresponding to the three basic kinds of ship motions: rectilinear motion $\left(\Omega_{1}\right)$, left and right turns $\left(\Omega_{2}\right.$ and $\left.\Omega_{3}\right)$ :

$$
\Omega=\left[\Omega_{1}, \Omega_{2}, \Omega_{3}\right]^{\prime}=[0, U,-U]^{\prime},
$$

where $U$ denotes the preset constant rate of turn. The vector $\Omega$ covers all possible ship manoeuvres and system noises in the band $[U,-U]$. The particular choice of $U$ is made by taking into account general considerations from the marine practice and some important international navigation restrictions. ${ }^{20}$

* The attempt to introduce respective vector of possible ship lengths has been recognised in ${ }^{17}$ as unsuccessful because of bad distinction of the resulting models. The uncertainty, concerning the ship geometry has been overcome by introducing a constant average ship length $l=$ const.$^{17}$

So, the final version of the requested ship model takes the following general form:

$$
x_{i, k+1}=f\left(x_{k}, \Omega_{i}, l\right), i=1,2,3,
$$

where $x_{k}=\left[X_{k}, Y_{k}, \psi_{k}, V_{0, k}\right]^{\prime}$ and where:

$$
\begin{aligned}
& X_{i, k+1}=X_{i, k}+T V_{i, k+1} \sin \psi_{i, k}, \\
& Y_{i, k+1}=Y_{i, k}+T V_{i, k+1} \cos \psi_{i, k}, \\
& \psi_{i, k+1}=\psi_{i, k}+T V_{i, k+1} \Omega_{i}, \\
& V_{i, k+1}=K_{V, i} V_{0, k},
\end{aligned}
$$


where

$$
K_{V, i}=\left(1+1.9 \Omega_{i}^{2} l^{2}\right)^{-1}, \quad \Omega=\left[\Omega_{1}, \Omega_{2}, \Omega_{3}\right]^{\prime}=[0, U,-U]^{\prime}
$$

Another model, based, on the extended state vector $x_{i, k}^{e}=\left[X_{i, k}, Y_{i, k}, \psi_{i, k}, V_{i, k}, \Delta \Omega_{i, k}\right]^{\prime}$ is suggested in ${ }^{18}$. The corresponding extended ship models $(i=1,2,3)$ have the form:

$$
\begin{aligned}
& X_{i, k+1}=X_{i, k}+T V_{i, k+1} \sin \psi_{i, k}, \\
& Y_{i, k+1}=Y_{i, k}+T V_{i, k+1} \cos \psi_{i, k}, \\
& \psi_{i, k+1}=\psi_{i, k}+T V_{i, k+1}\left(\Omega_{i}+\Delta \Omega_{i, k}\right), \\
& V_{i, k+1}=K_{V, i} V_{0, k}, \\
& \Delta \Omega_{i, k+1}=\Delta \Omega_{i, k},
\end{aligned}
$$

where $K_{V, i}=\left(1+1.9 \Omega_{i}^{2} l^{2}\right)^{-1}$. It takes into account possible differences $\Delta \Omega_{i, k}$ between the unknown true value of the ship rate of turn $\Omega_{k}$ and its values $\Omega_{i}$ fixed in the IMM algorithm. The influence of $\Delta \Omega_{i, k}$ on the velocity is not taken into account because of its insignificance.

\section{IMM algorithm for tracking of manoeuvring ship}

Models (12)-(15) and (16)-(20) are expanded in ${ }^{17}$ in Taylor time-series up to firstorder terms around the estimated state vector. They are used in an Extended Kalman Filters (EKF) and respective IMM algorithms. The IMM algorithm based on model (12)-(15) is denoted as IMM-A and the proposed IMM algorithm based on model (16)-(20) is denoted as IMM-B.

The measurement equation has the form:

$$
y_{k}=H x_{k}+w_{k},
$$

where $H$ is the measurement matrix,

$$
H=\left[\begin{array}{llll}
1 & 0 & 0 & 0 \\
0 & 1 & 0 & 0
\end{array}\right],
$$


$w_{k}$ is a white Gaussian measurement noise with covariance matrix $R_{k}$.

For convenience, the polar measurements "range-bearing" $y_{k}=\left[r_{k}, \beta_{k}\right]^{\prime}$, are transformed here in Cartesian ones:

$$
X_{k}=r_{k} \sin \beta_{k}, Y_{k}=r_{k} \cos \beta_{k} .
$$

So, the measurement vector acquires the new form $y_{k}=\left[X_{k}, Y_{k}\right]^{\prime}$. Respectively, the covariance matrix of the measurement errors is ${ }^{8}$ :

$$
R_{i, k}=\left[\begin{array}{cc}
\sigma_{r}^{2} \sin ^{2} \beta_{k}+r_{k}^{2} \sigma_{\beta}^{2} \cos ^{2} \beta_{k} & \left(\sigma_{r}^{2}-r_{k}^{2} \sigma_{\beta}^{2}\right) \sin \beta_{k} \cos \beta_{k} \\
\left(\sigma_{r}^{2}-r_{k}^{2} \sigma_{\beta}^{2}\right) \sin \beta_{k} \cos \beta_{k} & \sigma_{r}^{2} \cos ^{2} \beta_{k}+r_{k}^{2} \sigma_{\beta}^{2} \sin ^{2} \beta_{k}
\end{array}\right],
$$

where $\sigma_{r}$ and $\sigma_{\beta}$ are the standard deviations of the range and bearing angle.

The equations of the $i^{\text {th }}(i=1,2,3) \mathrm{EKF}$ are:

$$
\begin{aligned}
& \hat{x}_{i, k / k}=\hat{x}_{i, k / k-1}+K_{i, k} \gamma_{i, k} \\
& \hat{x}_{i, k+1 / k}=f\left(\hat{x}_{i, k / k}, \Omega_{i,} l\right), \\
& \gamma_{i, k}=y_{k}-H_{i} \hat{x}_{i, k / k-1}, \\
& P_{i, k / k-1}=\phi f_{i}^{x} P_{i, k-1 / k-1}\left(f_{i}^{x}\right)^{\prime}, \\
& S_{i, k}=H_{i} P_{i, k / k-1} H_{i}^{\prime}+R_{i, k}, \\
& K_{i, k}=P_{i, k / k-1} H_{i}^{\prime} S_{i, k}^{-1}, \\
& P_{i, k / k}=P_{i, k / k-1}-K_{i, k} S_{i, k} K_{i, k}^{\prime} .
\end{aligned}
$$

Here, $\hat{x}_{i, k / k}$ and $\hat{x}_{i, k+1 / k}$ are the filtered estimate of the state $x_{k}$ and its one-step prediction; $\gamma_{i, k}$ and $S_{i, k}$ are the filter residual process and its covariance matrix, $P_{i, k / k}$ is the error covariance matrix, $K_{i, k}$ is the filter gain matrix, $\phi \geq 1$ is the fudge factor.

The Jacobi matrix $f_{i}^{x}=\left.\frac{\partial_{i}}{\partial x_{i}}\right|_{x_{i}=\hat{x}_{i}}$ computed based upon the model (12)-(15) has the
form: 


$$
f_{i}^{x}=\left[\begin{array}{cccc}
1 & 0 & T \hat{V}_{i, k+1} \cos \hat{\psi}_{i, k} & T K_{V, i} \sin \hat{\psi}_{i, k} \\
0 & 1 & -T \hat{V}_{i, k+1} \sin \hat{\psi}_{i, k} & T K_{V, i} \cos \hat{\psi}_{i, k} \\
0 & 0 & 1 & T K_{V, i} \Omega_{i} \\
0 & 0 & 0 & K_{V, i}
\end{array}\right]
$$

the one based on model (16)-(20) is:

$$
f_{i}^{x}=\left[\begin{array}{ccccc}
1 & 0 & T \hat{V}_{i, k+1} \cos \hat{\psi}_{i, k} & T K_{V, i} \sin \hat{\psi}_{i, k} & 0 \\
0 & 1 & -T \hat{V}_{i, k+1} \sin \hat{\psi}_{i, k} & T K_{V, i} \cos \hat{\psi}_{i, k} & 0 \\
0 & 0 & 1 & T K_{V, i}\left(\Omega_{i}+\Delta \hat{\Omega}_{i, k}\right) & T \hat{V}_{i, k+1} \\
0 & 0 & 0 & K_{V, i} & 0 \\
0 & 0 & 0 & 0 & 1
\end{array}\right] .
$$

A hard logic is introduced in both IMM algorithms to avoid undesired combination of the estimates $\hat{V}_{1, k}, \hat{V}_{2, k}$ and $\hat{V}_{3, k}{ }^{17}$ :

$$
\begin{gathered}
\hat{V}_{i, k}=\hat{V}_{1, k}, \quad(i=2,3) ; \\
\hat{V}_{k}=\hat{V}_{1, k}, \quad \text { if } \mu_{1, k}>0.5,
\end{gathered}
$$

where $\mu_{i, k}$ is the probability of the event: "the $i^{\text {th }}$ model is topical at time $k$ ", $\hat{V}_{k}$ is the overall estimate of the ship velocity.

\section{Performance evaluation}

The performance of both IMM algorithms is compared by Monte Carlo simulations. ${ }^{2}$ Results for 100 independent runs, each one lasting 200 scans $(600 s, T=3 s)$ are given.

The simulation parameters of the true model (7)-(11) are standard ${ }^{20,17}: q_{21}=0.331$, $r_{21}=-0.629, \quad s_{21}=-0.104, \quad h_{1}=3.5, q_{31}=-4.64, \quad r_{31}=3.88, s_{31}=-1.019, L=99 m$, $\delta_{\text {min }}=3^{\circ}, \delta_{\max }=30^{\circ}$. The chosen initial conditions are: $X_{0}=Y_{0}=10000 \mathrm{~m}$, $\psi_{0}=45^{\circ}, V_{0}=30 \mathrm{~m} / \mathrm{s}$. Initially the ship moves rectilinearly. The applied pulsewise rudder angle control law is:

$$
\delta=\left\{\begin{array}{ll}
\delta_{\text {max }}, & k \in[51,67] \\
0, & k \notin[51,67]
\end{array} .\right.
$$


The true ship trajectory is presented in Fig.1.

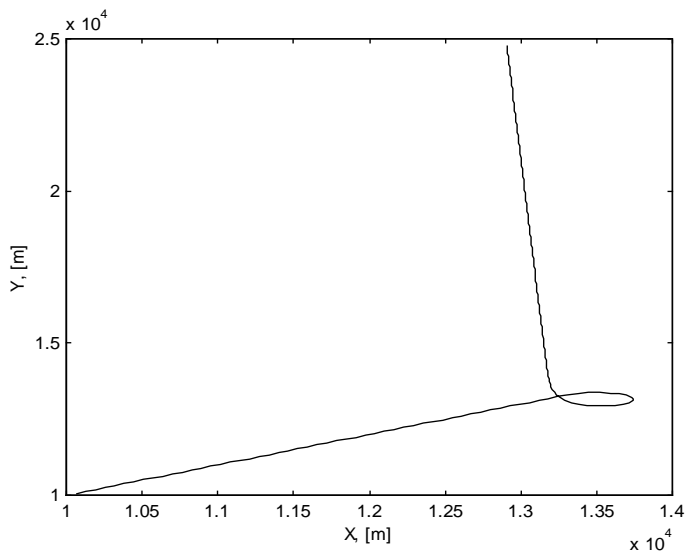

Figure 1: The true ship trajectory

Both considered IMM algorithms a use constant ship length $l=69 \mathrm{~m}$ for each of the three models, control parameter $U=0.0066 \mathrm{~m}^{-1}\left(360^{\circ} / \mathrm{min}\right)$ and fudge factors $\phi^{A}=\phi^{B}=1.03$. To compute the measurement error covariance matrix, it is preset: $\sigma_{r}=100 \mathrm{~m}, \sigma_{\beta}=0.3^{\circ}$. The initial error covariance matrices $P_{i, 0}$, the initial mode probability vectors $\mu$ and the transition probability matrices $\operatorname{Pr}$ are chosen as follows:

$$
\begin{aligned}
& P_{i, 0}^{A}=\operatorname{diag}\left\{\begin{array}{llll}
\sigma_{X}^{2} & \sigma_{Y}^{2} & \sigma_{\psi}^{2} & \sigma_{V}^{2}
\end{array}\right\}, \quad P_{i, 0}^{B}=\operatorname{diag}\left\{\begin{array}{lllll}
\sigma_{X}^{2} & \sigma_{Y}^{2} & \sigma_{\psi}^{2} & \sigma_{V}^{2} & \sigma_{\Delta \Omega}^{2}
\end{array}\right\}, \\
& \mu^{A}=\mu^{B}=\left[\begin{array}{c}
0.95 \\
0.025 \\
0.025
\end{array}\right], \operatorname{Pr}^{A}=\operatorname{Pr}^{B}=\left[\begin{array}{ccc}
0.6 & 0.2 & 0.2 \\
0.5 & 0.5 & 0 \\
0.5 & 0 & 0.5
\end{array}\right] . \\
& \sigma_{X}=\sigma_{Y}=\sigma_{r}, \sigma_{\psi}=0.1^{\circ}, \sigma_{V}=10 \mathrm{~m}, \sigma_{\Delta \Omega}=0.01 \mathrm{rad} / \mathrm{m} \text {. }
\end{aligned}
$$

The Monte Carlo simulation results are shown in Figs. 2-13. General estimation of the algorithms' performance is given in Fig.2. The IMM-B algorithm possesses better consistency during the manoeuvring stage.

These inferences are confirmed by results received for the mean error $(M E)$ and the root mean square errors (RMSE) of the state vector ${ }^{2}$ (Figs.3-6 and Figs.7-10). The 
average mode probabilities are presented in Figs.11-12. The computed ME of the estimated IMM-B control parameter change is given in Fig.13.

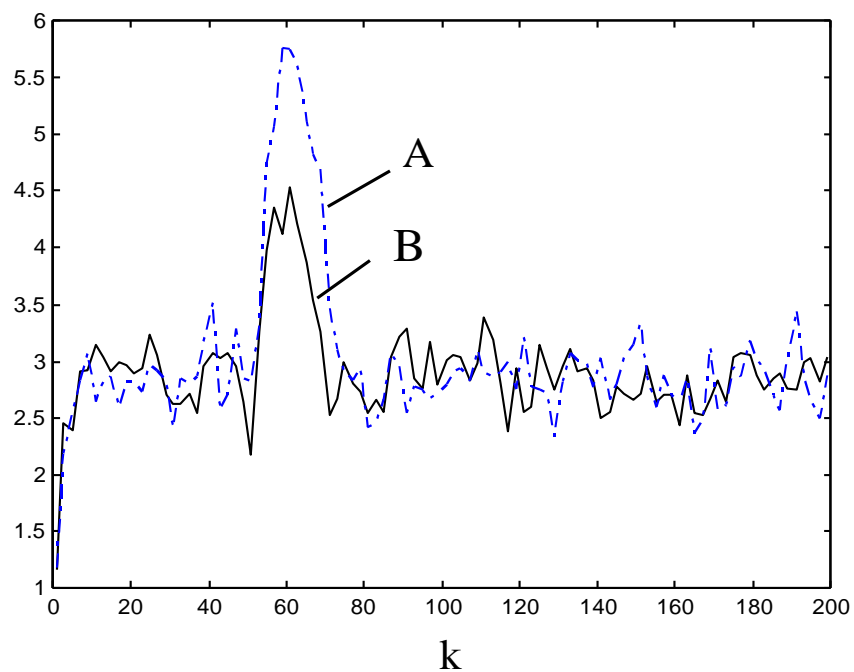

Figure 2: Normalized Estimation Error Squared

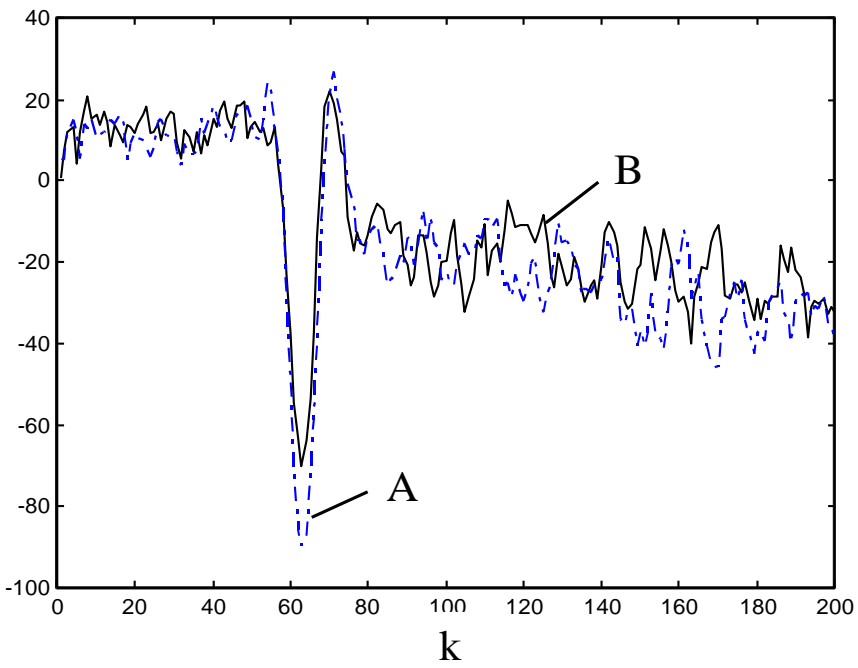

Figure 3: X Position ME 


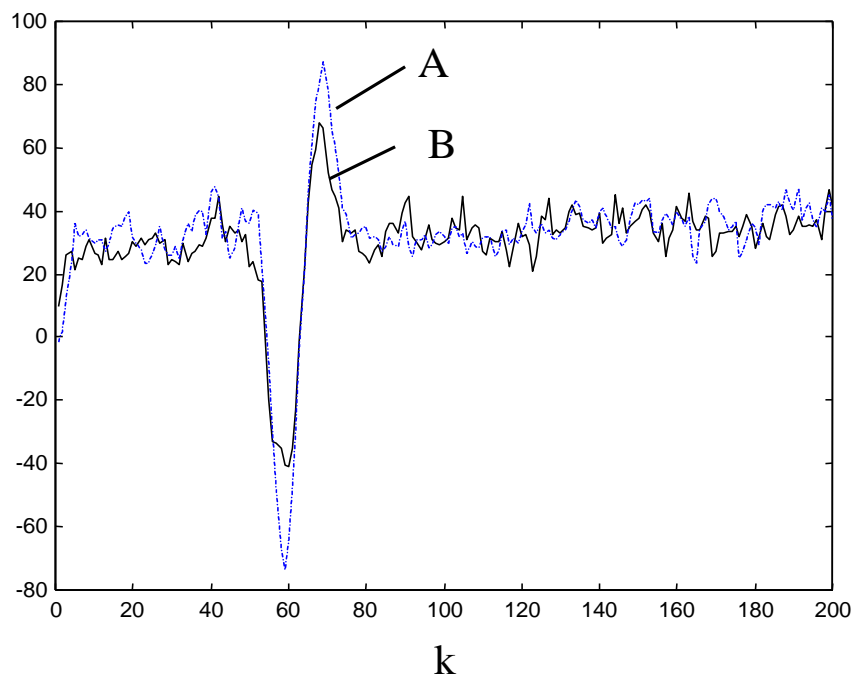

Figure 4: Y Position ME

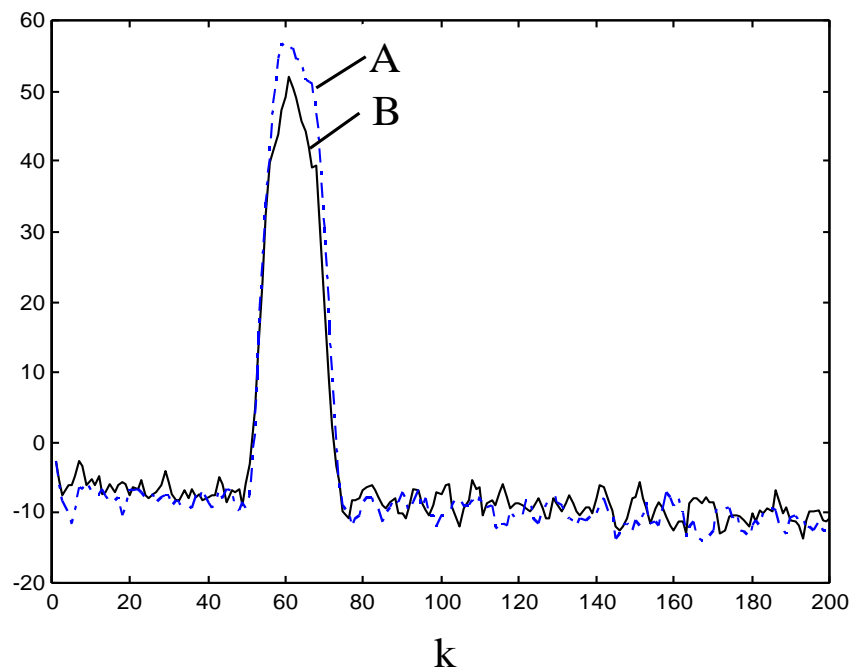

Figure 5: Heading ME 


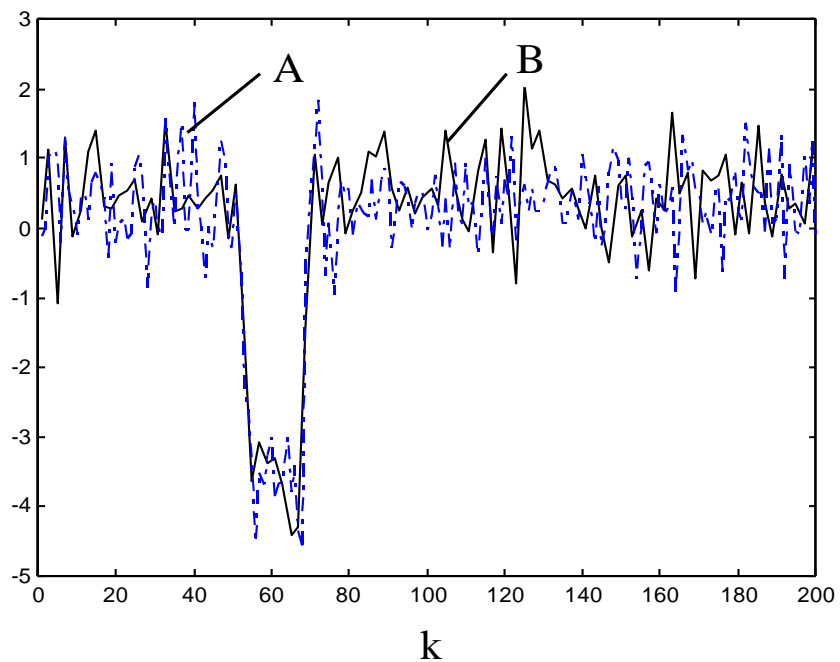

Figure 6: Velocity ME

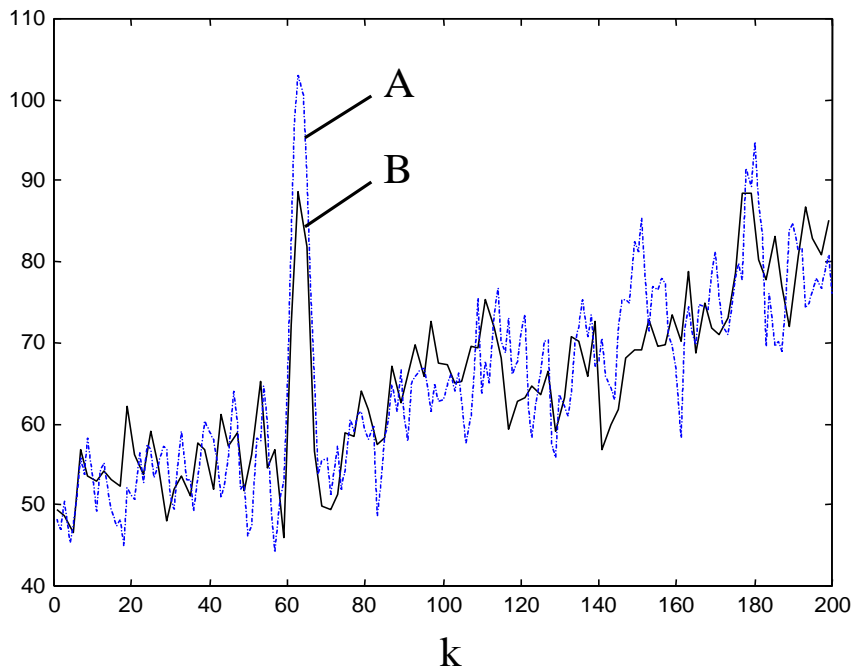

Figure 7: X Position RMSE 


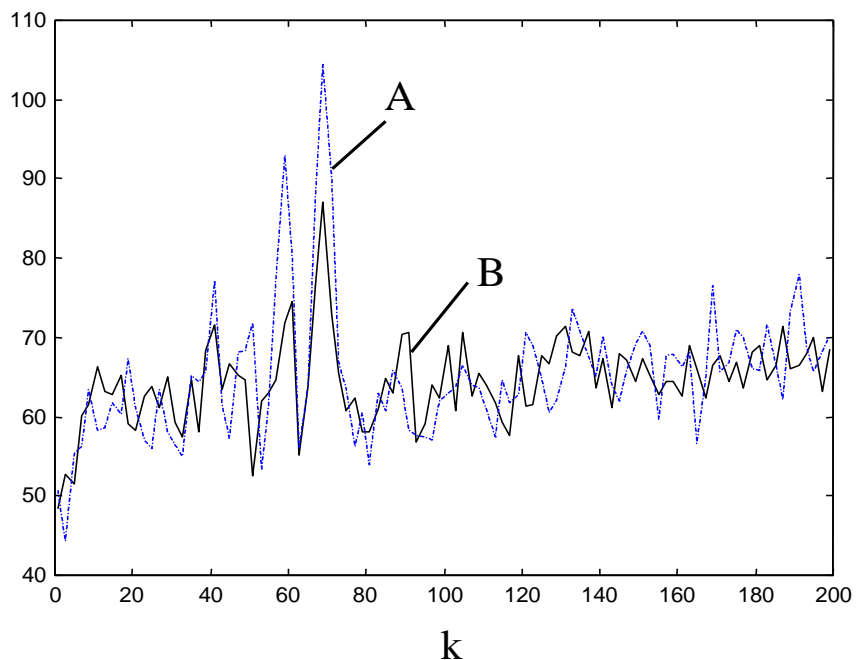

Figure 8: Y Position RMSE

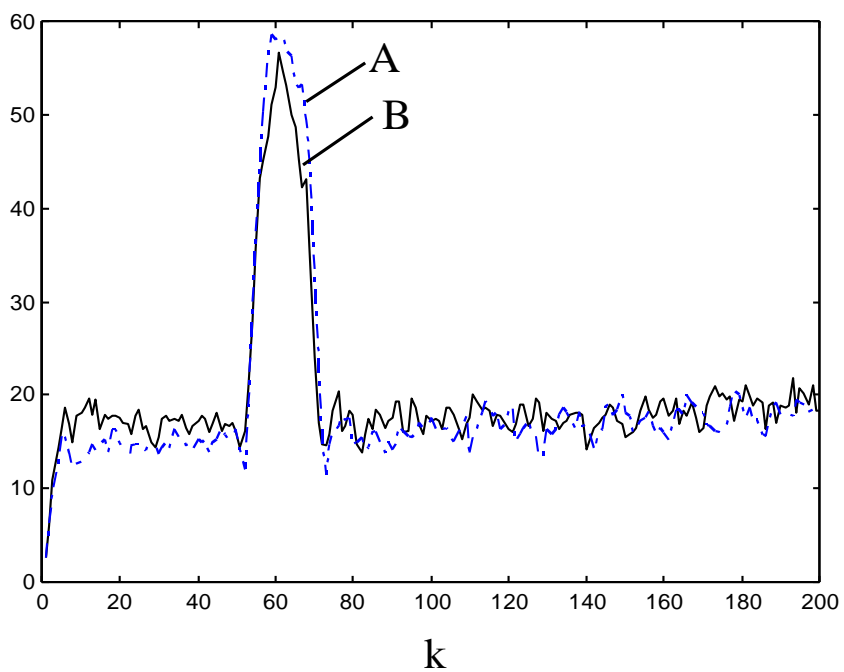

Figure 9: Heading RMSE 


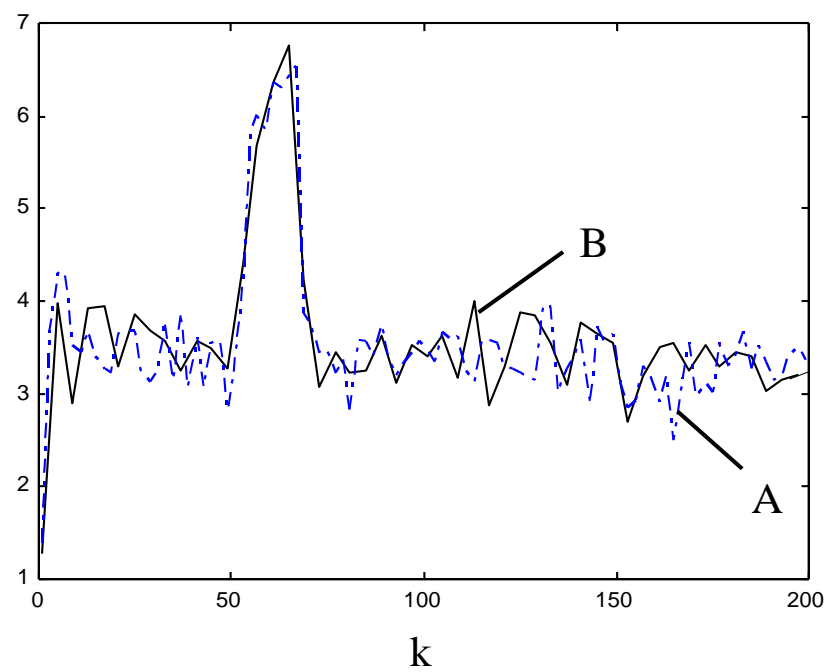

Figure 10: Velocity RMSE

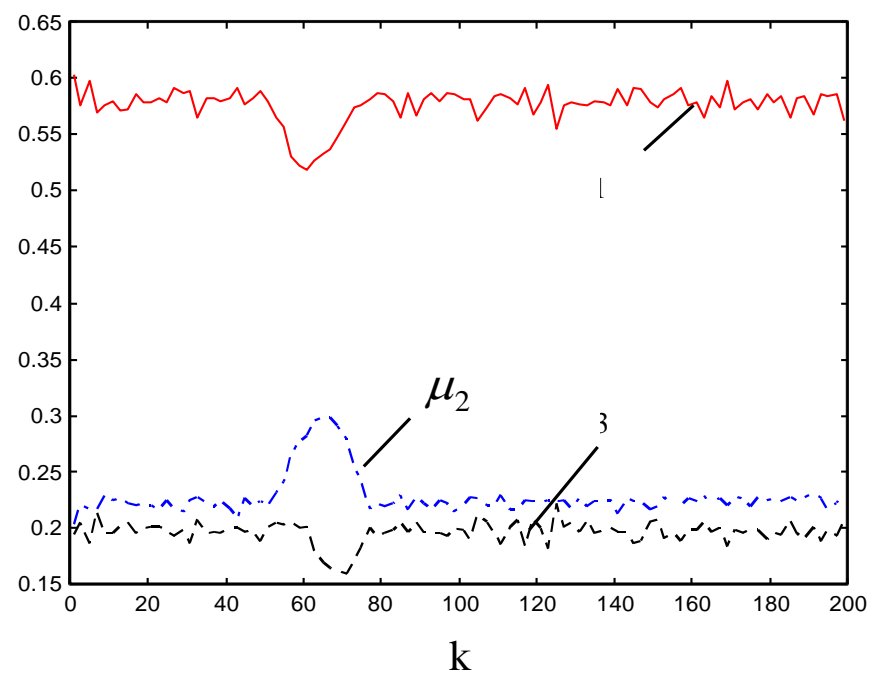

Figure 11: Average Mode Probabilities for IMM-A 


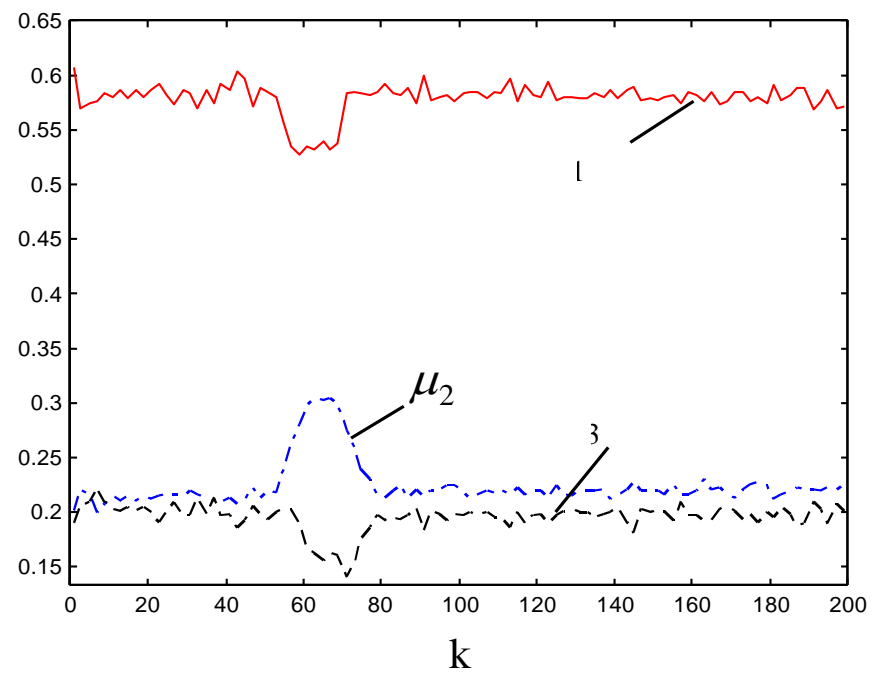

Figure 12: Average Mode Probabilities for IMM-B

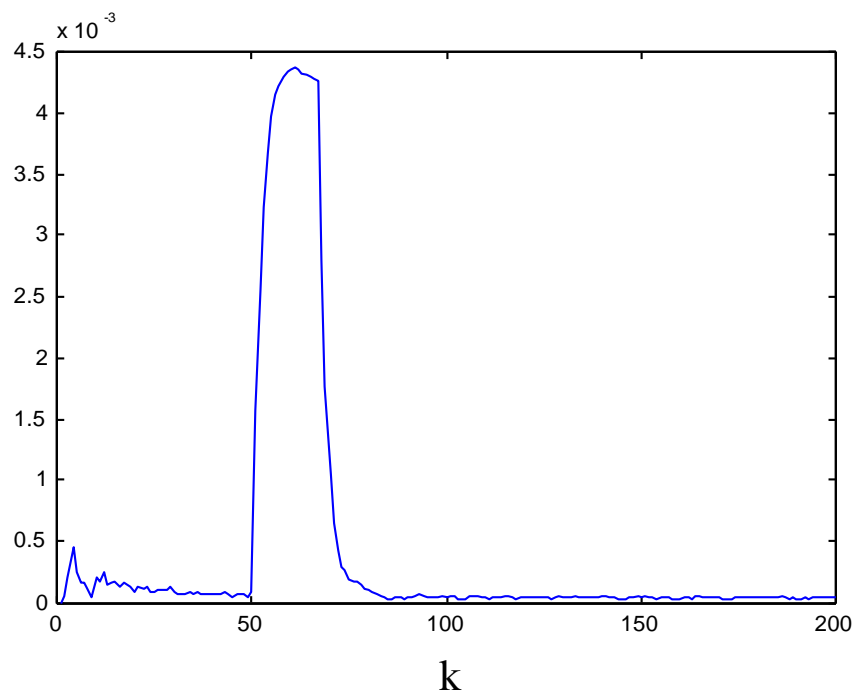

Figure 13: IMM-B control parameter change ME 


\section{Conclusions}

New models adequately describing the non-linear dynamics of maneuvering ship motion are proposed in the paper for manoeuvring ship tracking. They are implemented in a standard and in newly designed IMM versions. The proposed new IMM uses extended state vector and model to compensate the difference between the fixed control parameter of the currently used IMM model and its real value. The performed Monte Carlo simulations show excellent model fit and estimation performance.

Acknowledgement: The work on this paper was partially supported by contract No.I-808/98 with the Bulgarian Science Fund.

\section{References}

1. Abkowitz, M., Lectures on Ship Hydrodynamics - Steering and Maneuverability, HyA Rep.5 (1964).

2. Bar-Shalom, Y., ed., Multitarget-Multisensor Tracking: Applications and Advances, Vol. II (Artech House, 1992).

3. Bar-Shalom, Y. and Xiao Rong-Li, Estimation and Tracking Principles, Techniques and Software (Artech House, 1993).

4. Bar-Shalom, Y. and Xiao Rong-Li, Multitarget-Multisensor Tracking: Principles and Techniques (Storrs, CT: YBS Publ., 1995).

5. Best, R. and J. Norton, "A New Model and Efficient Tracker for a Target with Curvilinear Motion," IEEE Trans. on AES 33, 3 (1997), 1030-1037.

6. Blom H.A.P. and Y. Bar-Shalom, "The Interacting Multiple Model Algorithm for Systems with Markovian Switching Coefficients," IEEE Trans. on Automatic Control 33, 8 (1988), 780-783.

7. Ermolaev, G., A Handbook of the Captain Sailing at Far Distances (Moscow, Transport, 1981).

8. Farina, A. and F. Studer, Radar Data Processing, vol. I (J. Wiley \& Sons, 1986).

9. Lerro, D. and Y. Bar-Shalom, "Interacting Multiple Model Tracking with Target Amplitude Feature," IEEE Trans. on AES 29, 2 (1993), 494-508.

10. Xiao Rong-Li, "Hybrid Estimation Techniques," in Control and Dynamic Systems, vol.76, ed. C.T. Leondes (Academic Press, Inc., 1996), 213-287.

11. Mazor, E., A. Averbuch, Y. Bar-Shalom and J. Dayan, "IMM Methods in Target Tracking: A Survey," IEEE Trans. AES 34, 1 (1998), 103-123.

12. Nomoto, K., "Directional Steerability of Automatically Steered Ship with Particular Reference to Their Bad Performance in Rough See," DTMB Rep. 461, First Symposium on Ship Maneuverability (1960). 
13. Norrbin, N., "Theory and Observations on the Use of ARPA Mathematical Model for Ship Maneuvering in Deep and Confined Waters," SSPA 68 (1981).

14. Ogawa, A. and I. Kayama, "MMG Report I, On Mathematical Model of Maneuvering Motion of Ships," Bulletin of the SNAJ 575 (1977).

15. Pershitz, R., Ships Maneuverability and Control (Leningrad: Sudostroenie, 1973).

16. Emil Semerdjiev and Violeta Bogdanova, "Nonlinear Model and IMM Tracking Algorithm for Sea Radars," in Proc. of 40 Internationales Wissentschaftliches Kolloquim (Ilmenau, Germany: 1995), Band 1.

17. Emil Semerdjiev, Ludmila Mihaylova and Tzvetan Semerdjiev, "Maneuvering Ship Model Identification and IMM Tracking Algorithm Design," in Proceedings of the First International Conference on Multisource-Multisensor Information Fusion'98 (Las Vegas, Nevada: July 6-9, 1998), vol. 2, 968-973.

18. Emil Semerdjiev and Ludmila Mihaylova, "Adaptive IMM Algorithm for Maneuvering Ship Tracking," in Proceedings of the International Conference on MultisourceMultisensor Information Fusion'98, (Las Vegas, USA: July 6-9, 1998), vol. 2, 974-979.

19. Sobolev, G., Ship Maneuverability and Ships' Control Automation (Leningrad: Sudostroenie, 1976).

20. Voitkounski, Y., Ed., Ship Theory Handbook, Vol.1 (Leningrad: Sudostroenie, 1985).

EMIL ATANASOV SEMERDJIEV see page 68.

LUDMILA STOYANOVA MIHAYLOVA see page 68.

TZVETAN ATANASOV SEMERDJIEV see page 81.

VIOLETA GEORGIEVA BOGDANOVA is assistant research professor at the Central Laboratory for Parallel Processing, Bulgarian Academy of Sciences. She received M.S. degree in Sofia Technology Institute, in 1976. E-mail: signal@bas.bg. 